\title{
Old or Heavy? Decaying Gracefully with Age/Weight Shapes.
}

DOI:

10.1007/978-3-030-29436-6_27

\section{Document Version}

Accepted author manuscript

Link to publication record in Manchester Research Explorer

\section{Citation for published version (APA):}

Rawson, M., \& Reger, G. (2019). Old or Heavy? Decaying Gracefully with Age/Weight Shapes. In Automated Deduction - CADE 27 (Lecture Notes in Computer Science; Vol. 11716). https://doi.org/10.1007/978-3-030-294366_27

\section{Published in:}

Automated Deduction - CADE 27

\section{Citing this paper}

Please note that where the full-text provided on Manchester Research Explorer is the Author Accepted Manuscript or Proof version this may differ from the final Published version. If citing, it is advised that you check and use the publisher's definitive version.

\section{General rights}

Copyright and moral rights for the publications made accessible in the Research Explorer are retained by the authors and/or other copyright owners and it is a condition of accessing publications that users recognise and abide by the legal requirements associated with these rights.

\section{Takedown policy}

If you believe that this document breaches copyright please refer to the University of Manchester's Takedown Procedures [http://man.ac.uk/04Y6Bo] or contact uml.scholarlycommunications@manchester.ac.uk providing relevant details, so we can investigate your claim.

\section{OPEN ACCESS}




\title{
Old or Heavy? Decaying Gracefully with Age/Weight Shapes.
}

\author{
Michael Rawson and Giles Reger \\ University of Manchester, Manchester, UK
}

\begin{abstract}
Modern saturation theorem provers are based on the givenclause algorithm, which iteratively selects new clauses to process. This clause selection has a large impact on the performance of proof search and has been the subject of much folklore. The standard approach is to alternate between selecting the oldest clause and the lightest clause with a fixed, but configurable age/weight ratio (AWR). An optimal fixed value of this ratio is shown to produce proofs significantly more quickly on a given problem, and further that varying AWR during proof search can improve upon a fixed ratio. Several new modes for the Vampire prover which vary AWR according to a "shape" during proof search are developed based on these observations. The modes solve a number of new problems in the TPTP benchmark set.
\end{abstract}

\section{Introduction}

Currently, the most successful theorem provers (such as Vampire [4], E [12], and SPASS [17]) for first-order logic are saturation-based, utilising the wellknown given-clause algorithm. Simply, this algorithm saturates a set of clauses by iteratively selecting a clause and performing all non-redundant inferences with it until all clauses have been selected or the empty clause (witnessing inconsistency) has been found. Clearly, the order in which clauses are selected is key to the performance of the algorithm. Over the past few decades a certain amount of folklore has built up around the best methods for clause selection and recent work by Schulz and Möhrmann [13] systematically studied these. Our work extends this study with new results and also introduces the concept of a variable clause selection strategy (one that changes over time), instantiated with two simple patterns (or shapes) that prove to be pragmatically useful.

Clause selection strategies that alternate between selecting clauses based on age (i.e. in a first-in-first-out manner) and weight (i.e. those with the fewest symbols first) are the subject of this work. It was confirmed by Schulz and Möhrmann that alternating these two heuristics outperforms either by itself. The ratio of these selections is the age/weight ratio (AWR), as this is the terminology employed by the Vampire theorem prover, the vehicle for our study.

After covering relevant background material in Section 2 the remainder of the paper makes two main contributions. Firstly, Section 3 experimentally confirms the folklore that (i) the choice of age/weight ratio often has a significant effect on 
the performance of proof search, and (ii) there is no "best" age/weight ratio: indeed, a large range of pragmatically useful ratios exist. Section 4.1 demonstrates that varying the age-weight ratio over time can achieve better performance than a fixed ratio, and therefore motivates the addition of so-called age/weight shapes for varying the ratio over time. Experiments (Section 5) with these new options implemented in the Vampire theorem prover show a significant improvement in coverage, proving many new problems unsolvable by any previous configuration of Vampire.

\section{Background}

This section introduces the relevant background for the rest of the paper.

First-Order Logic and Weight. Our setting is the standard first-order predicate logic with equality. A formal definition of this logic is not required for this paper but an important notion is that of the weight of a clause. In first-order logic, terms are built from function symbols and variables, literals are built from terms, and clauses are disjunctions of literals. The weight of a term/literal is the number of symbols (function, variable, or predicate) occurring in it. The weight of a clause is the sum of the weights of its literals.

Saturation-Based Proof Search. Saturation-based theorem provers saturate a set of clauses $S$ with respect to an inference system $\mathbb{I}$ : that is, computing a set of clauses $S^{\prime}$ by applying rules in $\mathbb{I}$ to clauses in $S$ until no new clauses are generated. If the empty clause is generated then $S$ is unsatisfiable. Calculi such as resolution and superposition have conditions that ensure completeness, which means that a saturated set $S$ is satisfiable if it does not contain the empty clause as an element. As first-order logic is only semi-decidable, it is not necessarily the case that $S$ has a finite saturation, and even if it does it may be unachievable in practice using the available resources. Therefore, much effort in saturation-based first-order theorem proving involves controlling proof search to make finding the empty clause more likely (within reasonable resource bounds). One important notion is that of redundancy, being able to remove clauses from the search space that are not required. Another important notion are literal selections that place restrictions on the inferences that can be performed. Both notions come with additional requirements for completeness. Vampire often gives up these requirements for pragmatic reasons (incomplete strategies have been found to be more efficient than complete ones in certain cases) and in such cases the satisfiability of $S$ upon saturation is unknown.

The Given Clause Algorithm and AWR Clause Selection. To achieve saturation, the given clause algorithm organises the set of clauses into two sets: the active clauses are those that have been active in inferences, and the passive clauses are those that have not. Typically, a further unprocessed set is required in order to manage the clauses produced during a single iteration of the loop. Realisations of 
the given clause algorithm generally differ in how they organise simplifications. There are two main approaches (both implemented by Vampire, originally found in the eponymous theorem provers Otter [5] and Discount [1]): the Otter loop uses both active and passive for simplifications, whereas the Discount loop uses only active.

The algorithm is centred around the clause selection process. As previously mentioned, there are two main heuristics for this:

- By Age (or First-in/First-out) clause selection prefers the oldest clause (produced earlier in proof search), simulating a breadth-first search of the clause space. In Vampire the age of a clause is the number of inferences performed to produce it (input clauses have age 0 ).

- By Weight (or symbol-counting) clause selection prefers the lightest clause. The intuition behind this approach is that the sought empty clause has zero symbols and lighter clauses are in some sense closer to this. Furthermore, lighter clauses are more general in terms of subsumption and tend to have fewer children, making them less explosive in terms of proof search.

Schulz and Möhrmann show that alternating these heuristics is beneficial. In Vampire this alternation is achieved by an age/weight ratio (AWR) implemented by a simple balancing algorithm. The balance is initialised to 0 and used as follows: a negative balance means that a clause should be selected by age, whereas a positive balance means that a clause should be selected by weight; given a ratio of $a: w$ the balance is incremented by $a$ when selecting by age and decremented by $w$ when selecting by weight. Figure 1 gives the Discount algorithm along with balance-based AWR clause selection. The lines relevant to clause selection are marked with $\checkmark$.

Portfolio Solvers. Vampire is a portfolio solver and is typically run in a mode that attempts multiple different strategies in quick succession, e.g. in a 30 second run it may attempt 10 or more different strategies, and may run these in parallel with different priorities [8]. These strategies employ many different options including different saturation algorithms (including Otter and Discount), preprocessing options, literal selection strategies, inference rules, and clause selection heuristics. The portfolio mode is a significant improvement on any single strategy.

Vampire's portfolio mode also includes an additional option relevant to clause selection: the --nongoal_weight_coefficient option specifies a multiplier to apply to the weight of non-goal clauses, thus preferring clauses in or derived from the problem conjecture in clause selection. Use of this heuristic is orthogonal to the age/weight ratio and is not investigated further here.

Related Work. Many clause selection approaches are taken by other solvers. Otter $3.3[6]$ selects either by age, by weight or manually. Prover9 [7] allows a configurable age/weight ratio. E [12] allows the user to specify an arbitrary number of priority queues and a weighted round-robin scheme that determines how many clauses are picked from each queue. The default is to use a combination of age and weight selection, although there is also a complex strategy developed 


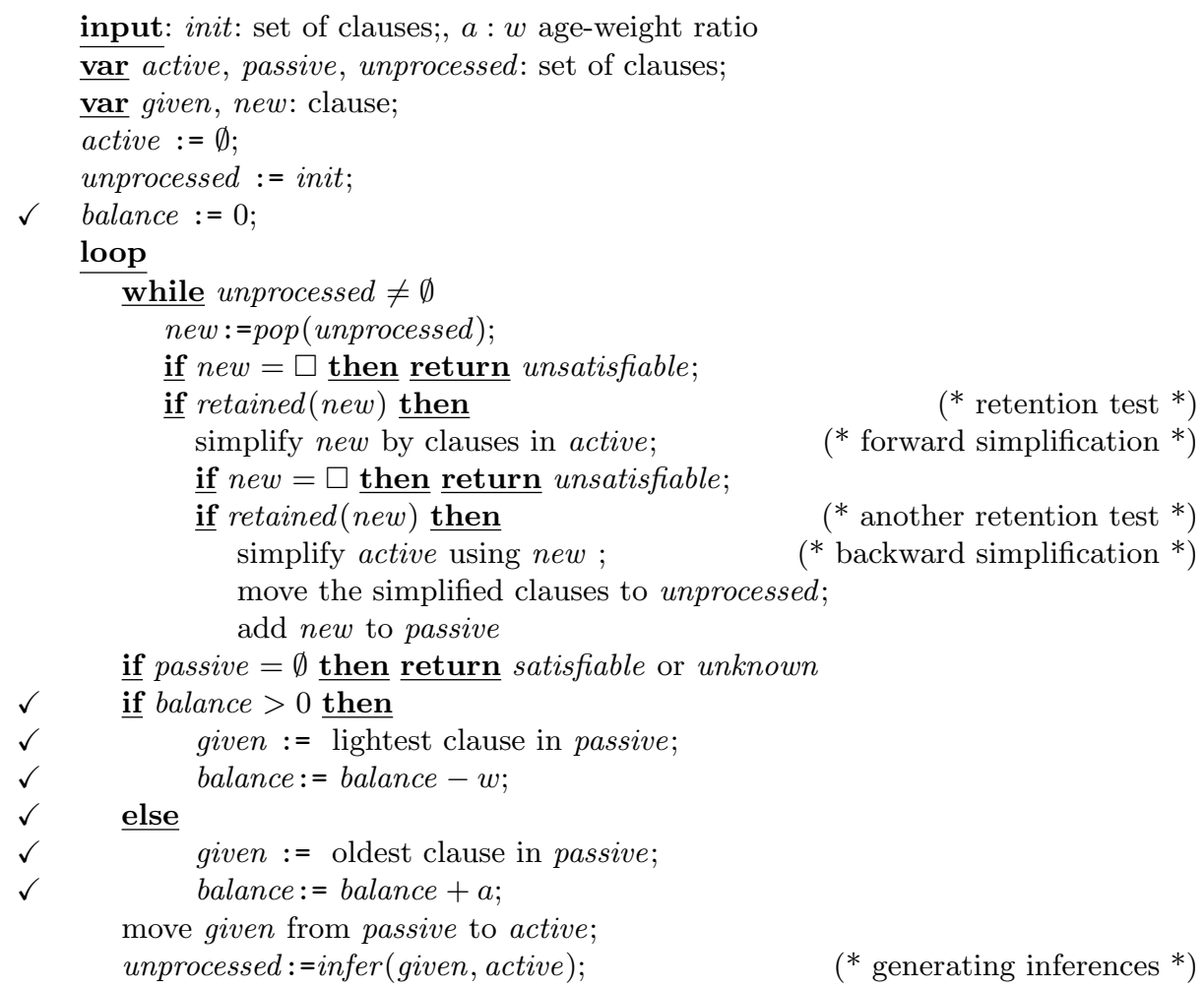

Fig. 1. The Discount Saturation Algorithm with AWR clause selection

by a genetic algorithm [11]. SPASS [17] uses symbol-counting based clause selection. iProver [3] follows $\mathrm{E}$ in having a number of configurable queues but relies mainly on age and weight heuristics in those queues. The general idea in this paper of a varying age/weight ratio over time is applicable to any ratio-based clause selection strategy, and our specific results apply to those that take a ratio between age and weight.

\section{Optimising Age/Weight Ratios}

Two assumptions from folklore are confirmed experimentally:

1. The choice of age/weight ratio often has a significant effect on the performance of proof search.

2. There is in general no single best age/weight ratio for a given set of problems.

These are supported by the work of Schulz and Möhrmann but are explored in more depth here. 


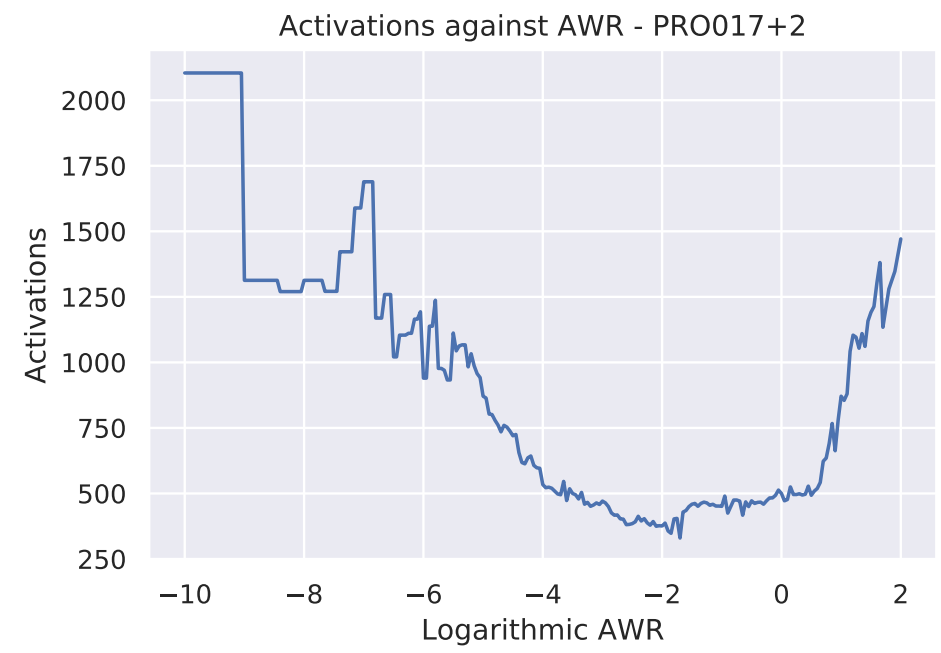

Fig. 2. The number of given-clause loops reported by Vampire after finding a proof with 1-second runs on a TPTP problem, PR0017+2. In between the peaks on either side, the function of $L$ is discontinuous with large peaks and troughs, but follows an approximate trend and settles toward the global optimum. PR0017+2 exhibits typifying behaviour for TPTP, based on manual inspection of several hundred such plots.

\subsection{Logarithmic AWR}

Visualising AWR values is more easily achieved if they have a continuous scale. AWR values are mathematically $\mathbb{Q}^{+}$, the positive rational numbers, but in practice are more easily visualised logarithmically. Therefore, the logarithmic AWR $L$ is defined in terms of age $A$ and weight $W$ as

$$
L=\log _{2}\left(\frac{A}{W}\right)
$$

As $L$ tends to positive infinity, Vampire selects only by weight, whereas if $L$ tends to negative infinity Vampire selects only by age. $L=0$ represents the middle ground of a 1:1 age/weight ratio. Note that the balancing algorithm used by Vampire does not make use of this value (it still requires two numbers) but the quantity is used in this work to show continuous AWR values.

\section{$3.2 \quad$ Experiments}

As an initial illustrative example of how varying the AWR effects the number of clauses required to be processed before a proof is found consider Figure 2. This demonstrates the effect that varying AWR can have: a smaller number of activations means that fewer clauses were processed, which in general means 
Table 1. Relative performance gain, showing the ratio in activations between the best AWR setting for a given problem and another base setting. A comparison is drawn between 1:1 (Vampire's default), 1:5 (the best-behaved from Schulz and Möhrmann), and the worst setting for the problem. Where the problem is not solved at all by the base setting, it is ignored.

\begin{tabular}{c|c|cc} 
Base Setting & $\%$ Maximum Gain & $\%$ Mean Gain (Standard Deviation) \\
\hline $1: 1$ & 13,356 & 126 & 163 \\
$1: 5$ & 13,367 & 144 & 170 \\
(worst) & 22,201 & 395 & 760
\end{tabular}

that a proof was found faster ${ }^{1}$. On the problem shown, a good AWR value is over $400 \%$ better by this metric than the worst AWR value.

This experiment was repeated on the whole TPTP problem set, excluding problems Vampire does not currently support (e.g. higher-order problems). Vampire ran for 1 second in default mode with the discount saturation algorithm ${ }^{2}$ using a sensible set of AWR values (see Table 2) - these are the values used in Vampire's portfolio mode. These tend to favour weight-first over age-first as this has been experimentally shown to be preferable. Problems not solved by any of these, or those solved trivially (e.g in preprocessing) are removed. The whole set yielded data for 7,947 problems.

The first result is that choosing a good AWR value for a problem is wellrewarded. Table 1 summarises the impact that choosing the best AWR can have. Compared to the default, Vampire can perform, on average, 1.26 times fewer activations, which is modest but (as Table 2 shows) just under $10 \%$ of problems are no longer proven by choosing the default. It is more relevant to note that there are cases where Vampire can do much better by selecting a different AWR value. Therefore, choosing a better AWR value can go from no solution to a solution and can do so faster, but not necessarily. In the worst case (choosing the pessimal AWR value) Vampire performs almost 4 times as many activations.

The second result is that there is no "best" AWR across this full set of problems. Drop in performance is defined to be how many times more activations were required for a proof under a given AWR, compared to the best AWR. Table 2 shows, for each AWR value, the \% of problems solved, the number solved uniquely, and the maximum and mean drop in performance. No AWR value solves all problems, with the best being 1:5. A ratio of 1:4 produces an unusually small maximum performance drop. Schulz and Möhrmann found that 1:5 had a similar property, but this might be explained by differences in prover and test environment. It is interesting to note that the extreme AWR values solve fewer

\footnotetext{
${ }^{1}$ It should be noted that if a small number of clauses are extremely expensive to process it may be slower than a larger number of less-expensive clauses, but in general this is a good heuristic measure for prover performance. It also avoids reproducibility issues involved with using system timing approaches.

${ }^{2}$ The default LRS [10] saturation algorithm can be non-deterministic.
} 
Table 2. Per-AWR value results on 1 second runs over 7,947 TPTP problems.

\begin{tabular}{|c|c|c|c|c|c|}
\hline AWR & $\%$ Solved & |Uniques & \% Maximum Drop & \% Mean Drop & (Standard Deviation) \\
\hline $8: 1$ & 85.25 & 16 & 15,067 & 137 & 198 \\
\hline $5: 1$ & 86.10 & 1 & 12,222 & 133 & 164 \\
\hline $4: 1$ & 86.93 & 1 & 10,144 & 132 & 142 \\
\hline $3: 1$ & 87.63 & 2 & 10,500 & 129 & 141 \\
\hline $2: 1$ & 88.62 & 3 & 11,267 & 127 & 145 \\
\hline $3: 2$ & 89.83 & 2 & 11,989 & 127 & 151 \\
\hline $5: 4$ & 89.98 & 4 & 12,500 & 126 & 155 \\
\hline $1: 1$ & 90.56 & 4 & 13,356 & 126 & 163 \\
\hline $2: 3$ & 91.20 & 9 & 14,767 & 128 & 179 \\
\hline $1: 2$ & 91.68 & 0 & 16,267 & 131 & 197 \\
\hline $1: 3$ & 91.81 & 5 & 19,056 & 137 & 230 \\
\hline $1: 4$ & 91.85 & 3 & 1,741 & 138 & 67 \\
\hline $1: 5$ & 92.00 & 2 & 13,367 & 144 & 170 \\
\hline $1: 6$ & 91.57 & 1 & 10,644 & 147 & 146 \\
\hline $1: 7$ & 91.49 & 1 & 10,489 & 149 & 144 \\
\hline $1: 8$ & 91.09 & 2 & 10,133 & 153 & 145 \\
\hline $1: 10$ & 90.52 & 1 & 10,178 & 160 & 153 \\
\hline $1: 12$ & 90.00 & 0 & 10,167 & 165 & 162 \\
\hline $1: 14$ & 89.29 & 4 & 10,300 & 170 & 175 \\
\hline $1: 16$ & 89.42 & 5 & 10,133 & 174 & 176 \\
\hline $1: 20$ & 88.61 & 3 & 10,089 & 182 & 194 \\
\hline $1: 24$ & 88.26 & 2 & 10,133 & 189 & 208 \\
\hline $1: 28$ & 87.57 & 2 & 9,922 & 196 & 224 \\
\hline $1: 32$ & 87.01 & 1 & 10,000 & 199 & 236 \\
\hline $1: 40$ & 86.23 & 4 & 9,878 & 209 & 264 \\
\hline $1: 50$ & 84.93 & 1 & 9,878 & 217 & 288 \\
\hline $1: 64$ & 84.17 & 2 & 10,122 & 228 & 319 \\
\hline $1: 128$ & 81.34 & 3 & 10,744 & 257 & 416 \\
\hline $1: 1024$ & 73.11 & 23 & 22,201 & 283 & 755 \\
\hline
\end{tabular}

problems overall but solve the most uniquely. This is typical in saturation-based proof search: approaches that do not perform well in general may perform well in specific cases where the general approach does not.

In summary, these results confirm the previous assumptions often made in folklore. It should be noted that this is a small experiment (1 second runs in default mode) and the relative performance of different AWR values cannot be generalised, but the general result that they are complementary can.

\section{Variable AWR for Vampire}

This section motivates and defines a clause selection approach which varies the AWR value over time. 


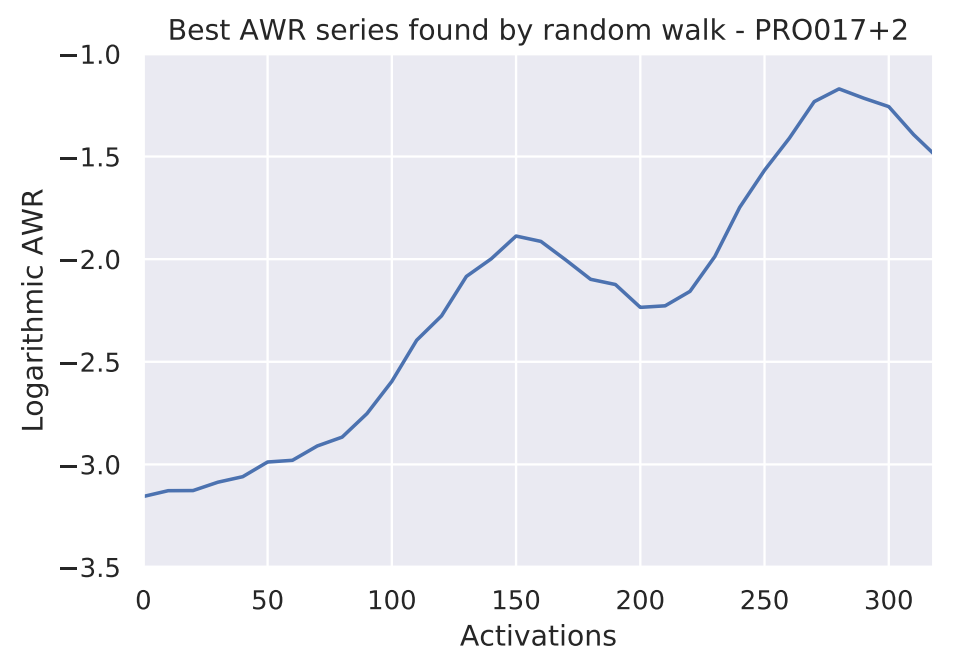

Fig. 3. The AWR series that produced the lowest number of activations on a particular problem, smoothed in order to show the actual effect on proof search. This is a search strategy that a single fixed AWR cannot reproduce.

\subsection{The Optimal AWR Over Time}

Although choosing a good AWR value is important, this is covered in part by the use of strategy scheduling in which many AWR values are tried in sequence (along with other prover options). Additionally, given that varying the AWR can have such a large impact, it seems likely that a constant AWR fixed for the entire proof search is unlikely to be optimal for any given problem. This can be shown by running Vampire with a randomised sequence of age/weight ratios given by a random walk repeatedly, then finding the best after a large number of repetitions. Applying this method with 10,000 repetitions to the problem seen earlier $($ PRO017+2) yields the example AWR trend shown in Figure 3, which reduces the best number of activations from 330 with a fixed AWR, to 287 with a varying AWR. Unsurprisingly, in ad-hoc experiments on other problems, the best shape is rarely constant. This suggests that implementing other shapes, such as an increasing or decreasing trend, might lead to quicker proofs in the Vampire theorem prover.

\subsection{Varying AWR (in Vampire)}

An implementation of dynamically-varying AWR values in Vampire is described below. In general any possible sequence that the AWR could follow during proof search can be used. However, some details constrain the design space:

1. Changing the AWR too frequently or sharply has little effect, due to the "balancing" algorithm — see Section 1. 

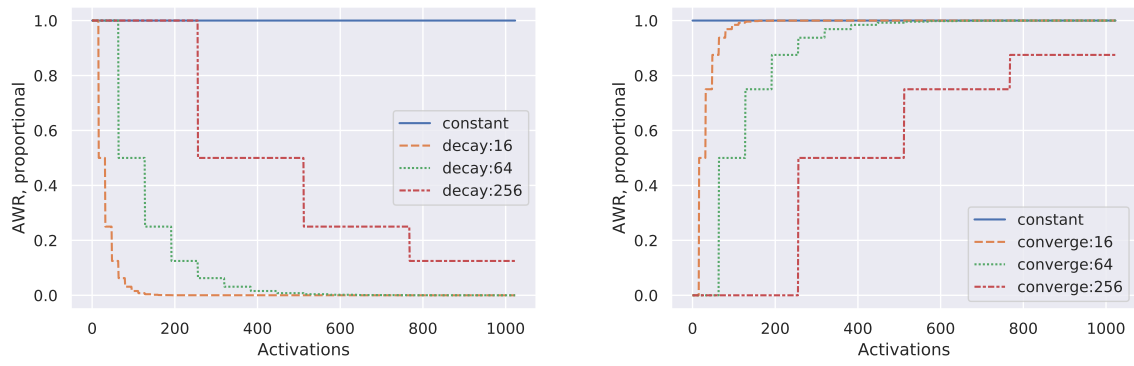

Fig. 4. The new decay and converge AWR shapes as implemented in Vampire. Different curves exhibit the effect of the AWR shape frequency setting.

2. A general (configurable) shape is more likely to be widely applicable than a specific series of data points.

3. The shape must extend naturally to an indefinitely-long proof search.

In this work two general trends are explored: a trend away from a given start AWR toward 1:1 ("decay"), and a trend from 1:1 toward a given end AWR ("converge"). Investigation showed that even fluctuating sequences had a general trend, and further that these two fixed trends are reasonable approximations of these trends. The start/end AWR values are taken from the portfolio mode: these values are known to be useful in a fixed-AWR context, and while this may not generalise to a dynamic-AWR context, it is a useful starting point pending integration of AWR shape parameters into strategy scheduling.

Since a simple linear shape does not extend well to indefinite proof search (it is unclear what should happen after either 1:1 or the target AWR is reached), an exponential decay function is used instead. These exponential shapes are further parameterised by an integral shape frequency setting, which controls the rate of decay or convergence: every $n$ steps, the difference between the current and the target AWR is halved, rounding where necessary. In future, this might allow the use of repeating patterns such as a sinusoid, hence frequency. Figure 4 illustrates rates at which the new configurations converge or decay from the fixed AWR setting for some indicative frequency settings.

Our approach here was restricted by the balancing algorithm used internally, as AWR steps must be discrete and do not take effect immediately. An alternative approach might be to use an age/weight probability, rather than a ratio, from which age or weight decisions would be pseudo-randomly (but reproducibly) taken with the use of a seeded pseudo-random number generator, permitting use of continuous age/weight functions.

Two new options are implemented:--age_weight_ratio_shape can take the values constant, decay, or converge and selects one of the above shapes; and --age_weight_ratio_shape_frequency specifies the frequency (rate) or convergence/decay (default is 100). These are used with the existing--age_weight_ratio 
option (default 1:1) to give a number of new option combinations, which can be used in conjunction with Vampire's portfolio mode pending integration into the strategy schedules. This version of the prover is currently in a separate branch in the main Vampire source repository ${ }^{3}$. Another option, --age_weight_ratio_b is implemented (default 1:1), controlling the initial AWR value of converge or the final AWR value of decay.

\section{Experimental Evaluation}

Two experiments evaluate the new techniques. The first compares the various options attempting to draw some conclusions about which option values work well together. The second looks at how useful the new options are in the context of portfolio solving. Both experiments use the TPTP (version 7.1.0) benchmark [16] and were run on StarExec [14].

\subsection{Comparing New Options}

Vampire ran in default mode (with the discount saturation algorithm) for 10s whilst varying age_weight_ratio and age_weight_ratio_shape_frequency for several AWR shapes: constant, converging from 1:1, decaying to 1:1, converging from 1:4 and decaying from 1:4.

Results are given in Table 4 . The results for the different shapes are grouped into columns and then by frequency with rows giving results per AWR value. The total number of problems solved and those solved uniquely are also reported. The best combination of options overall was decaying from an initial age/weight of 1:100 with frequency 1000. Longer frequencies tended to do better, suggesting that more time at the intermediate AWR values is preferable. Unique solutions are distributed well in general, showing that the new options are complementary.

\subsection{Contribution to Portfolio}

Our next experiment aims to answer the question "How much can the portfolio mode of Vampire be improved using these new options?". To address this the new options ran on top of the portfolio mode used in the most recent CASC competition CASC-J9 [15]. Note that the CASC-J9 portfolio mode contains techniques completely unrelated to the age/weight ratio, e.g. finite model building [9], as well as other options related to clause selection, e.g. non-goal weight coefficient and set-of-support.

Vampire first ran to establish baseline performance in the given portfolio mode on all problems in TPTP, with a wallclock time limit of 300 seconds. New options were applied on top of the portfolio mode options, using the existing AWR values in the various strategies as the starting point. Three shapes are employed: constant (baseline), converging from 1:1 and decaying to 1:1. The

\footnotetext{
${ }^{3}$ https://github.com/vprover/vampire/tree/awr-shapes
} 
Table 3. Results for the tested configurations. Proved refers to the total number of problems a configuration solved. Fresh is the number of problems a configuration solved which were not solved by the baseline. Uniques is the number of problems a configuration solved which were not solved by any other configuration. $u$-score is a refined unique score which correlates to a configuration's utility in solving new problems, as used in Hoder et al. [2].

\begin{tabular}{|c|c|c|c|c|c|}
\hline Configuration & Frequency & Proved & Fresh & Uniques & $u$-score \\
\hline baseline & - & 13,057 & & 1 & 714.2 \\
\hline converge & 1 & 13,039 & 24 & 3 & 714.3 \\
\hline converge & 5 & 13,029 & 27 & 1 & 709.5 \\
\hline converge & 10 & 13,028 & 35 & 5 & 714.3 \\
\hline converge & 50 & 13,015 & 45 & 5 & 712.8 \\
\hline converge & 100 & 12,976 & 51 & 1 & 705.9 \\
\hline converge & 500 & 12,895 & 63 & 4 & 698.3 \\
\hline converge & 1000 & 12,837 & 52 & 0 & 688.6 \\
\hline converge & 5000 & 12,775 & 53 & 1 & 682.4 \\
\hline converge & 10000 & 12,751 & 53 & 0 & 678.7 \\
\hline decay & 1 & 12,698 & 48 & 1 & 673.6 \\
\hline decay & 5 & 12,702 & 51 & 1 & 674.9 \\
\hline decay & 10 & 12,698 & 48 & 1 & 674.2 \\
\hline decay & 50 & 12,712 & 49 & 2 & 679.1 \\
\hline decay & 100 & 12,726 & 46 & 1 & 678.8 \\
\hline decay & 500 & 12,795 & 29 & 1 & 685.5 \\
\hline decay & 1000 & 12,860 & 29 & 2 & 692.6 \\
\hline decay & 5000 & 12,982 & 16 & 2 & 707.1 \\
\hline decay & 10000 & 13,002 & 7 & 0 & 706.3 \\
\hline converge & (combined) & 13,167 & 117 & 41 & - \\
\hline decay & (combined) & 13,106 & 93 & 17 & - \\
\hline
\end{tabular}


Table 4. Number of problems solved (top) and unique problems solved (bottom) by various configurations varying start/end AWR values, AWR shape, and AWR frequency. Bold numbers indicate the best result within a given shape.

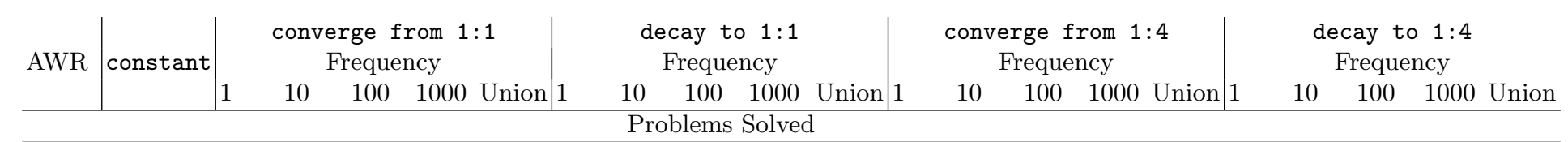

\begin{tabular}{l|l|l|llll|lllll|llllll|llll}
\hline $10: 1$ & 7967 & 7972 & 7976 & 8050 & 8245 & 8372 & 8448 & 8441 & 8380 & 8169 & 8614 & 7983 & 7990 & 8094 & 8323 & 8485 & 8579 & 8574 & 8493 & 8272 & 8797
\end{tabular}

\begin{tabular}{l|l|lllll|llllllllllllllllll}
$1: 10$ & 8575 & 8565 & $\mathbf{8 5 7 8}$ & 8550 & 8489 & 8778 & 8458 & 8456 & 8484 & 8268 & 8787 & 8575 & $\mathbf{8 5 8 4}$ & 8582 & 8535 & 8729 & 8584 & 8574 & 8590 & 8608 & 8764
\end{tabular}

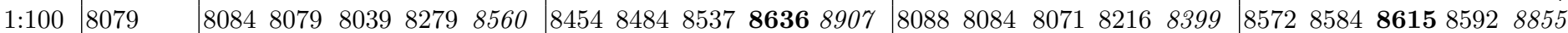

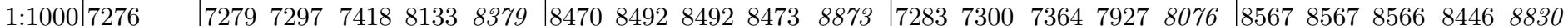

\begin{tabular}{l|l|llllllllllllllllllll}
\hline Union & 9019 & 9028 & 9016 & 8981 & 8871 & 9194 & 8572 & 8674 & 8725 & 8978 & 9048 & 9038 & 9038 & 9016 & 8967 & 9180 & 8697 & 8759 & 8800 & 8927 & 9026 \\
\hline
\end{tabular}

\begin{tabular}{|c|c|c|c|c|c|c|c|c|c|c|c|c|c|c|c|c|c|c|c|c|c|}
\hline \multicolumn{22}{|c|}{ Uniquely Solved } \\
\hline $10: 1$ & 1 & 2 & 1 & 1 & 6 & 10 & 0 & 0 & 0 & 2 & 2 & 1 & 0 & 0 & 1 & 2 & 0 & 0 & 2 & 4 & 6 \\
\hline $1: 100$ & 1 & 0 & 0 & 5 & 4 & 9 & 2 & 0 & 3 & 3 & 8 & 0 & 0 & 0 & 2 & 2 & 0 & 1 & 1 & 0 & 2 \\
\hline Union & 2 & 3 & 1 & 6 & 12 & 22 & 4 & 0 & 3 & 13 & 20 & 1 & 2 & 3 & 6 & 12 & 0 & 1 & 3 & 4 & 8 \\
\hline
\end{tabular}


Table 5. Total number of problems solved compared to other solvers.

\begin{tabular}{llll} 
Solver & Total solved & \multicolumn{2}{c}{$\begin{array}{c}\text { Uniquely solved } \\
\text { Excluding Vampire (old) }\end{array}$} \\
\hline Vampire (old) & 13,057 & 0 & - \\
Vampire (new) & 13,191 & 54 & 1030 \\
E & 10,845 & 190 & 190 \\
iProver & 8,143 & 215 & 215 \\
CVC4 & 9,354 & 501 & 502
\end{tabular}

purpose is to gauge what impact adding such options to a new portfolio mode could have. In this experiment the aim was to find new solved problems and identify new strategies that could be added to a portfolio mode. Therefore, it makes sense to consider the union of all experiments.

Overall, the baseline solved the most problems (13,057). No experimental configuration improved on this figure, but some problems not solved by baseline were solved by the new configurations, and some entirely new problems were solved. The union of all converge and decay configurations improved on the baseline, with 13,167 and 13,106 solved problems respectively.

Figure 3 shows the performance in terms of solved problems of all the configurations tested. These data show that configurations which select clauses in a similar way to the baseline (i.e. slow decay or fast convergence) achieve similar performance, as expected. In total, $134(117+17,93+41)$ problems were solved by the new configurations that were not solved by the baseline. This is an impressive result - it is rare to be able to improve portfolio mode by this many new problems with a single new proof search option.

The $u$-score is computed by giving $1 / n$ points per problem solved where $n$ is the number of strategies solving a problem [2]. This gives a measure of contribution per strategy. Options with the largest $u$-score will be prioritised for extending the existing portfolio mode, but only those with unique solutions overall.

Finally, two problems were solved which were marked with an "Unknown" status (with rating 1.00) in the TPTP headers. Only converging with frequency 50 solved SET345-6 and only decaying with frequency 1 solved LAT320+3.

\subsection{Comparison with other Solvers}

To place these results in context, the overall number of problems solved by our new strategies are compared with the results of other solvers, using their CASCJ9 These results are from 300-second runs in identical conditions and are given in Table 5. In this table Vampire (old) stands for the CASC-J9 competition version whilst Vampire (new) stands for the union of all problems solved by new options in the previous section. Between them, the two versions of Vampire solve 1,030 problems uniquely. 54 unique problems found in the previous section remain unique when compared to other competitive solvers. 


\section{Conclusions and Future Work}

Clause selection is a key part of any saturation-based theorem prover and age/weight ratios have a significant effect on the performance of proof search in the Vampire theorem prover. Known folklore that there is no clear optimal age/weight ratio is supported. Further, varying the age/weight ratio over time during proof search can improve further on an optimal, but fixed age/weight ratio in terms of the number of activations. Experiments within Vampire on the TPTP benchmark set suggest that these age/weight shapes show promise for future developments in this novel approach to proof search. Indeed, including our relatively simple shapes already leads to significant performance gains.

Future directions for research include trying a greater number of "shapes" (such as repeating patterns), other approaches for parameterising these shapes, a pseudo-random approach to age/weight instead of the balancing algorithm, and integration of the new approaches into existing strategy schedules.

\section{References}

1. Jörg Denzinger, Martin Kronenburg, and Stephan Schulz. Discount-a distributed and learning equational prover. Journal of Automated Reasoning, 18(2):189-198, 1997.

2. Kryštof Hoder, Giles Reger, Martin Suda, and Andrei Voronkov. Selecting the selection. In International Joint Conference on Automated Reasoning, pages 313329. Springer, 2016.

3. Konstantin Korovin. iProver - an instantiation-based theorem prover for first-order logic (system description). In Armando, Baumgartner, and Dowek, editors, IJCAR 2008, volume 5195 of Lecture Notes in Computer Science, pages 292-298, 2008.

4. Laura Kovács and Andrei Voronkov. First-order theorem proving and Vampire. In International Conference on Computer Aided Verification, pages 1-35. Springer, 2013.

5. William McCune. Otter 2.0. In International Conference on Automated Deduction, pages 663-664. Springer, 1990.

6. William McCune. Otter 3.3 reference manual. arXiv preprint cs/0310056, 2003.

7. William McCune. Release of prover9. In Mile High Conference on Quasigroups, Loops and Nonassociative Systems, Denver, Colorado, 2005.

8. Michael Rawson and Giles Reger. Dynamic strategy priority: Empower the strong and abandon the weak. In Proceedings of the 6th Workshop on Practical Aspects of Automated Reasoning co-located with Federated Logic Conference 2018 (FLoC 2018), Oxford, UK, July 19th, 2018., pages 58-71, 2018.

9. Giles Reger, Martin Suda, and Andrei Voronkov. Finding finite models in multisorted first-order logic. In Theory and Applications of Satisfiability Testing - SAT 2016 - 19th International Conference, Bordeaux, France, July 5-8, 2016, Proceedings, pages 323-341, 2016.

10. Alexandre Riazanov and Andrei Voronkov. Limited resource strategy in resolution theorem proving. 36(1-2):101-115, 2003.

11. Simon Schäfer and Stephan Schulz. Breeding theorem proving heuristics with genetic algorithms. In Global Conference on Artificial Intelligence, GCAI 2015, Tbilisi, Georgia, October 16-19, 2015, pages 263-274, 2015. 
12. Stephan Schulz. E - a brainiac theorem prover. 15(2-3):111-126, 2002.

13. Stephan Schulz and Martin Möhrmann. Performance of clause selection heuristics for saturation-based theorem proving. In Automated Reasoning - 8th International Joint Conference, IJCAR 2016, Coimbra, Portugal, June 27 - July 2, 2016, Proceedings, pages 330-345, 2016.

14. Aaron Stump, Geoff Sutcliffe, and Cesare Tinelli. StarExec: a cross-community infrastructure for logic solving. In International joint conference on automated reasoning, pages 367-373. Springer, 2014.

15. Geoff Sutcliffe. The 9th ijcar automated theorem proving system competitioncasc-j9. AI Communications, (Preprint):1-13, 2015.

16. Geoff Sutcliffe. The TPTP problem library and associated infrastructure, from CNF to TH0, TPTP v6.4.0. Journal of Automated Reasoning, 59(4):483-502, 2017.

17. Christoph Weidenbach. Combining superposition, sorts and splitting. In Robinson and Voronkov, editors, Handbook of Automated Reasoning, volume II, chapter 27, pages 1965-2013. Elsevier Science, 2001. 